\title{
The Effect of Backbone Extension in Thienobenzo[b]indacenodithiophene Polymers for Organic Field-Effect Transistors
}

\author{
Hu Chen ${ }^{*}$, Andrew Wadsworth ${ }^{*} \|$, Chun $\mathrm{Ma}^{\dagger}$, Alice Nanni ${ }^{\dagger}$, Weimin Zhang ${ }^{\dagger}$, Mark Nikolka ${ }^{\ddagger}$, Al- \\ exander M. T. Luci ${ }^{\S}$, Luís M. A. Perdigão§, Karl J. Thorley॥, Camila Cendra ${ }^{\circledR}$, Bryon Larson ${ }^{\gamma}$, Garry \\ Rumbles $^{\gamma}$, Thomas D. Anthopoulos ${ }^{\dagger}$, Alberto Salleo ${ }^{\perp}$, Giovanni Costantini ${ }^{\S}$, Henning Sirringhaus \\ ", and Iain McCulloch ${ }^{\dagger} \|$ \\ ${ }^{\dagger}$ King Abdullah University of Science and Technology (KAUST), KAUST Solar Center (KSC), Thuwal 23955-69oo, \\ Saudi Arabia. \\ ${ }^{\ddagger}$ Optoelectronics Group, Cavendish Laboratory, University of Cambridge, JJ Thomson Avenue, Cambridge, CB3 \\ oHE, UK. \\ $\S$ Department of Chemistry University of Warwick, Gibbet Hill Road, Coventry CV4 7AL, UK. \\ ॥ Department of Chemistry and Centre for Plastic Electronics, Imperial College London, Exhibition Road, London, \\ $\mathrm{SW}_{7}$ 2AZ, United Kingdom. \\ $\gamma$ National Renewable Energy Laboratory, Golden, CO, 80401, USA. \\ $\perp$ Department of Materials Science and Engineering, Stanford University, 476 Lomita Mall, Stanford, CA 94305, \\ USA.
}

KEYWORDS: OFET, Mobility, IDT, TBIDT, STM, DFT.

\begin{abstract}
A fused donor, thienobenzo[b]indacenodithiophene (TBIDT), was designed and synthesized using a novel acid-promoted cascade ring closure strategy, and copolymerized with a benzothiadiazole (BT) monomer. The backbone of TBIDT is an extension of the well-known indacenodithiophene (IDT) unit and was expected to enhance the charge carrier mobility, by improving backbone planarity and facilitating short-contacts between polymer chains. However, the optimized field-effect transistors demonstrated an average saturation hole mobility of $0.9 \mathrm{~cm}^{2} \mathrm{~V}^{-1} \mathrm{~s}^{-1}$, lower than the performance of IDT-BT $\left(\sim 1.5 \mathrm{~cm}^{2} \mathrm{~V}^{-1} \mathrm{~s}^{-1}\right)$. Mobilities extracted from time-resolved microwave conductivity (TRMC) measurements were consistent with the trend in hole mobilities in OFET devices. Scanning Tunneling Microscopy (STM) measurements and computational modelling illustrated that TBIDT-BT exhibits a less ordered microstructure in comparison to IDT-BT. This reveals that a regular side chain packing density, independent of conformational isomers, is critical to avoid local free volume due to irregular packing, which can host trapping impurities. DFT calculations indicated that TBIDT-BT, despite containing a larger, planar unit, showed less stabilization of planar backbone geometries, in comparison to IDT-BT. This is due to the reduced electrostatic stabilizing interactions between the peripheral thiophene of the fused core with the BT unit, resulting in a reduction of the barrier to rotation around the single bond. These insights provide a greater understanding of the general structure-property relationships required for semiconducting polymer repeat units to ensure optimal backbone planarization, as illustrated with IDT-type units, guiding the design of novel semiconducting polymers with extended fused backbones for high-performance field-effect transistors.
\end{abstract}

\section{INTRODUCTION}

During the past decade, $\pi$-conjugated organic semiconductors have attracted substantial interest owing to advantages that they possess over their inorganic counterparts; these include their easily tunable optoelectronic and structural properties, lightweight nature, cost-effectiveness, mechanical robustness and the capability to fabricate large area devices..$^{1-5}$ The charge carrier mobility of organic field-effect transistors (OFETs), which is the most crucial parameter that determines the characteristics in such devices, has increased from less than $0.1 \mathrm{~cm}^{2} \mathrm{~V}^{-1} \mathrm{~s}^{-1}$ to exceed the benchmark of thin-film amorphous silicon devices $\left(0.5^{-1} \mathrm{~cm}^{2} \mathrm{~V}^{-1} \mathrm{~s}^{-1}\right)$, up to $>3 \mathrm{~cm}^{2} \mathrm{~V}^{-}$ ${ }^{1} \mathrm{~s}^{-1}$ for both polymers and small molecules. ${ }^{6,7} \mathrm{In}$ addition to developments in device architecture optimization, the structural design of organic semiconductors has become a key strategy to achieve excellent device performance. ${ }^{8-10}$ Polymers containing 4,9-dihydro-s-indaceno[1,2-b:5,6-b']dithiophene (IDT), a promising molecular design motif, are characterized by low energetic disorder, excellent solution processability and exhibit high charge carrier mobility in ptype devices. The unique IDT unit has a fused rigid-ring backbone, with two $\mathrm{sp}^{3}$ bridging carbons, substituted with aliphatic chains to confer its solubility, as well as acting as a template for molecular packing. It was first demonstrated as a building block for high-performance OFET polymers by copolymerization with 2,1,3-benzothiadiazole (BT), to produce IDT-BT."This is an amorphous p-type polymer with a hole mobility of up to $3.6 \mathrm{~cm}^{2} \mathrm{~V}^{-1} \mathrm{~s}^{-1} \cdot{ }^{10}$

Despite its relatively amorphous nature, IDT-BT possesses impressive p-type transport properties, owing to the high degree of backbone order along polymer chains. ${ }^{10}$ It has been suggested that the key interaction that imparts the backbone order upon IDT-BT is between the nitrogen of the BT group and the IDT hydrogen $\alpha$ to the IDT-BT linkage, via non-traditional hydrogen bonding, leading to a high energetic barrier to backbone torsion. ${ }^{12}$ Maintaining the thienyl-BT linkage in analogous OFET polymers is therefore a desirable strategy to encourage highly ordered polymer backbones in 
IDT polymers. Extension of the IDT core has previously been used, with the aim of increasing the short-contact interactions between polymer chains, in order to improve the charge carrier mobility. Thienoindenofluorene (TIF) was copolymerized with BT, resulting in the polymer TIF-BT, and was able to achieve a hole mobility of $2.9 \mathrm{~cm}^{2} \mathrm{~V}^{-1} \mathrm{~s}^{-1} \cdot{ }^{13}$ Notably, it was suggested that the extended TIF core improved the inter-chain charge transport. ${ }^{13,14}$ Despite this, only a few examples have been reported that describe modifications of the IDT unit, owing mainly to the complex synthesis of extended fused-ring systems.

\section{Scheme 1. The synthetic transformation of IDT to TBIDT}

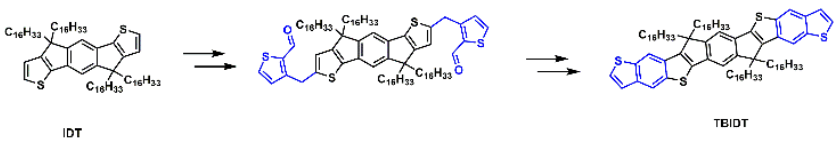

Herein, we have designed and reported the synthesis of a polymer comprising of a novel extended IDT unit, namely thiophenebenzo[b]indacenodithiophene (TBIDT), which was formed using an acid-promoted cascade ring closure strategy (Scheme 1). By synthesizing the aldehyde precursor, a cyclization/dehydration cascade reaction was utilized to afford the desired TBIDT monomer in a high yield. This novel synthetic strategy will prove to be useful in order to access other fused aromatic units for organic electronic applications. The TBIDT monomer was copolymerized with BT and the resulting polymer (TBIDT-BT) exhibited an average mobility of $0.9 \mathrm{~cm}^{2} \mathrm{~V}^{-1} \mathrm{~s}^{-1}$ in OFET devices

\section{RESULTS AND DISCUSSION}

\section{Scheme 2. Synthetic route to afford TBIDT-BT}

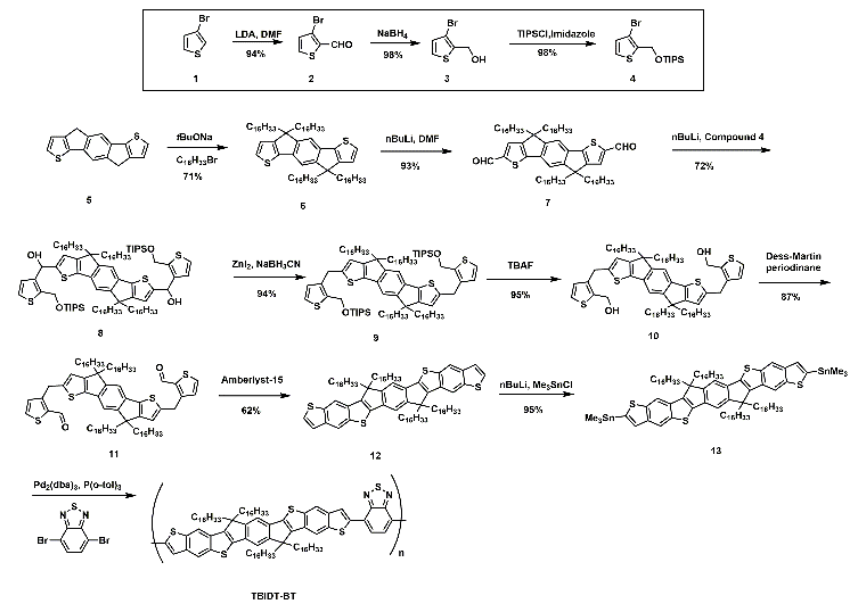

Scheme 2 shows the synthesis of the TBIDT-BT polymer. Firstly, it was necessary to synthesize the key intermediate (4) via four high yielding steps: the readily available 3-bromothiophene was selectively deprotonated at the 2 position and quenched by the addition of dimethyl formamide to produce 3-bromothiophene-2-carbaldehyde (compound 2), which was then reduced to (3-bromothiophen-2-yl)methanol (compound 3) by sodium borohydride. The triisopropylsilane group was found to be the best protecting group to protect the hydroxy group and afforded compound 4 . It should be noted that other commonly used protecting groups, such as trimethylsilane and triethylsilane, were shown to decompose during later steps of the TBIDT synthesis.

The next key intermediate to be prepared was the dialdehyde-IDT(compound 7). Alkylation of the known compound 4,9-dihydro-s-indaceno[1,2-b:5,6-b']dithiophene (compound 5) afforded the IDT monomer with $C_{1} 6$ alkyl chains (compound 6). Deprotonation of C16-IDT followed by quenching with dimethylformamide gave dialdehyde-IDT (compound 7) in high yield.

Having successfully produced the key intermediates (4 and 7), the next step was to construct the cyclization precursor (11). This first involved the conversion of compound 4 to a nucleophile, which was carried out by a halogen exchange step using butyl lithium. The in-situ generated nucleophile attacked the dialdehyde-IDT and afforded compound $\mathbf{8}$. To avoid the direct reaction of butyl lithium with compound 7 , it was necessary to use slightly fewer equivalents of butyl lithium than of compound $\mathbf{4}$ and the halogen exchange step was completed before the addition of the dialdehyde-IDT. In order to prepare compound $\mathbf{9}$, exhaustive screening and optimization of the reaction conditions was required. The combination of $\mathrm{NaCNBH}_{3} / \mathrm{ZnI}_{2}$ was found to be the mildest condition for the reduction of the hydroxyl groups, in high yield, without affecting other functional groups. Deprotection of 9 using TBAF then afforded compound 10, which was then oxidized to compound $\mathbf{1 1}$ using Dess-Martin Periodinane in a high yield. Dess-Martin Periodinane provided the best oxidation for the transformation, while other stronger oxidants such as chromium oxide also oxidized the $\mathrm{sp}^{3}$ carbons, affording relatively low yields.

Having successfully synthesized the cyclization precursor 11, the final step in producing the desired TBIDT motif was a cyclization/dehydration of compound 11. Different acids were screened to promote the cascade cyclization/dehydration. Amberlyst-15 was found to be the most efficient reagent to afford the desired product in moderate yield, in comparison to other acids such as trifluoroacetic acid, acetic acid and ptoluene sulfonic acid. This versatile procedure offers great potential to be utilized for the synthesis of other novel bridged donor molecules.

Upon completion of the novel fused aromatic unit, stannylation of TBIDT afforded the monomer 13. This was then copolymerized with dibromo-BT using microwave-assisted Stille conditions to produce the donor-acceptor (D-A) copolymer TBIDT-BT. Variation of the polymerization process resulted in an optimum number average molecular weight, determined by gel permeation chromatography (GPC), of $\sim 62$ $\mathrm{kDa}(\mathrm{Mn})$ with a very narrow polydispersity index (PDI) of 1.4, after purification using Soxhlet extraction and GPC. UV-Vis absorption spectra of the TBIDT-BT and its parent polymer IDT-BT are shown in Supplementary Information Figure $S_{7}$. Both the polymers showed two maxima peaks, with a shoulder close to the second peak, which is the common dual-band absorption observed for many donor-acceptor type polymers. TBIDT-BT shows a significant blue-shift in the onset of its absorption and its absorption maximum, compared to IDT-BT. This indicates that the extension of the backbone has resulted in an increase in the band gap of TBIDT-BT. The ionization potential (IP) of both polymers 
was measured by photoelectron spectroscopy in air (PESA) and the data shown in Table 1. The IP was found to be approximately $5.5 \mathrm{eV}$, which is slightly deeper than IDT-BT (5.4 $\mathrm{eV}$ ) due to the slightly lower electron density of the unit from a reduced thiophene ratio of aromatic groups. Conversely, the electron affinity (EA) of TBIDT-BT is less than IDT-BT due to the lower ratio of the electron withdrawing BT unit along the backbone.

Table 1. Summary of the Properties of the Polymers

\begin{tabular}{lccccc}
\hline Polymers & $\mathrm{Mn} / \mathrm{Mw}(\mathrm{kDa})^{\mathrm{a}}$ & $\lambda \mathrm{max} / \mathrm{nm}^{\mathrm{b}}$ & Eopt.gap $^{\mathrm{c}}(\mathrm{eV})$ & $\left.\mathrm{IP}^{(\mathrm{eV}}\right)^{\mathrm{d}}$ & $\mathrm{EA}(\mathrm{eV})^{\mathrm{e}}$ \\
\hline IDT-BT & $58.3 / 95.3$ & 666 & 1.7 & -5.4 & -3.7 \\
TBIDT- & $62.1 / 87.4$ & 595 & 1.9 & -5.5 & -3.6 \\
BT & & & & &
\end{tabular}

a Solution absorption spectra (in chlorobenzene), $\mathrm{M}_{\mathrm{n}}$ : molar mass averages of the number; $M_{w}$ : molar mass averages of the weight.

b Solid state absorption spectra from pristine thin-film.

c Obtained from the onset value of absorption in pristine thin-film. Eopt.gap: The gap between IP and EA.

d Measured by Photo-Electron Spectroscopy in Air (PESA)

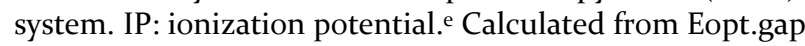
and IP. EA: electron affinity.

The thermal properties of both IDT-BT and TBIDT-BT thin films were investigated using thermogravimetric analysis (TGA) and differential scanning calorimetry (DSC), see supplementary information. Both polymers showed good thermal stability up to $400{ }^{\circ} \mathrm{C}$, while the DSC showed no discernible thermal transitions at temperatures up to $300{ }^{\circ} \mathrm{C}$. This is indicative of a low degree of ordering in the films. The observation of relatively disordered IDT-BT and TBIDT-BT thinfilms is also consistent with the Atomic Force Microscopy (AFM) measurements and the Grazing Incidence Wide-Angle X-ray Scattering (GIWAXS) data of both polymers (Figure 1).

a)

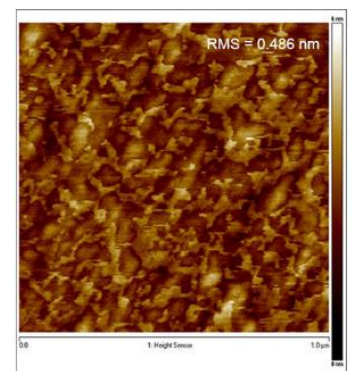

c)

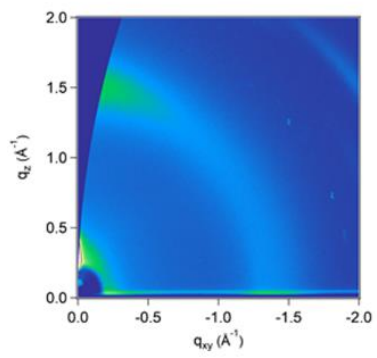

b)

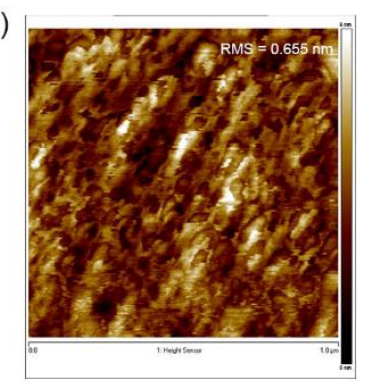

d)

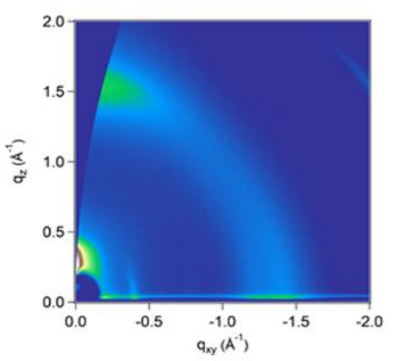

Figure 1. AFM images of: a) TBIDT-BT thin film; b) IDT-BT thin film. 2D GIWAXS patterns of c) TBIDT-BT thin film; d) IDT-BT thin film.

Tapping mode AFM was used to investigate the surface morphology of the polymer thin films (Figure 1a and Figure $1 b$ ). The surface roughness for the two polymers is comparable, with a root mean square roughness RMS $=0.486 \mathrm{~nm}$ and 0.655 $\mathrm{nm}$ extracted for TBIDT-BT and IDT-BT, respectively, consistent with a lack of crystalline texture. GIWAXS analysis was performed on as-cast films to provide insight into the thin film microstructure. Figure $1 \mathrm{c}$ and id show the two-dimensional GIWAXS patterns of TBIDT-BT and IDT-BT, respectively (see SI for the $1 \mathrm{D}$ lineouts taken along the out-of-plane and inplane scattering directions). A broad $\pi-\pi$ stacking peak is observed out-of-plane at a peak center position of $\mathrm{q}=1.49 \AA^{-1}$ and $\mathrm{q}=1.53 \AA^{-1}$ for TBIDT-BT and IDT-BT, respectively. This corresponds to $\pi-\pi$ stacking distances of $4.22 \AA$ and $4.10 \AA$ for TBIDT-BT and IDT-BT, respectively. Both polymers show relatively weak crystalline ordering, consistent with their predominantly amorphous nature, IDT-BT shows an in-plane peak at around $0.4 \AA^{-1}$, corresponding to the (oo1) plane of backbone stacking, while TBIDT-BT has no trace of this peak. This indicates better ordering of IDT-BT and it is in line with the STM observation.(Figure 4 ) The slightly shorter $\pi-\pi$ stacking distances in IDT-BT also suggest that it is able to pack more efficiently than TBIDT-BT.

Next, we evaluated the electrical performance and charge transport properties of TBIDT-BT and IDT-BT by fabricating bottom-contact top-gate (BC/TG) field effect transistors (see $\mathrm{SI}$ ). Figure $2 \mathrm{a}$ shows the representative transfer characteristics of TBIDT-BT OFETs with the linear and saturation characteristics scaling according to theory. Despite a delayed switchingon of the devices at $-3 \mathrm{~V}$ and a threshold voltage $\left(\mathrm{V}_{\mathrm{th}}\right)$ of $-15 \mathrm{~V}$, they exhibit a steep sub-threshold swing and an on-off ratio of $10^{6}$. Also, the output characteristics of a typical device shown in Figure $2 \mathrm{~b}$ do not present any pinching at lower gate- and drain-voltages suggesting that devices are not limited by poor injection. Subsequent extraction of the saturation charge carrier mobility of TBIDT-BT devices resulted in an average hole mobility of $0.9 \mathrm{~cm}^{2} \mathrm{~V}^{-1} \mathrm{~s}^{-1}$ across 8 devices, compared to $1.5 \mathrm{~cm}^{2}$ $\mathrm{V}^{-1} \mathrm{~S}^{-1}$ for IDT-BT measured in the same device architecture. It is well known that erroneous charge carrier mobility calculations, extracted from transistor current voltage characteristics, have at times led to an overestimate of mobility.15, 16 All charge carrier mobilities exhibited a pronounced gate-voltage $\left(\mathrm{V}_{\mathrm{G}}\right)$ dependence, with the highest values obtained only at $\mathrm{V}_{\mathrm{G}}$ $=-60 \mathrm{~V}$ (Figure 2c). A similar gate voltage dependence has been observed also for other polymers in the IDT-polymer family, such as TIF-BT ${ }^{13}$ but not in IDT-BT. ${ }^{11}$ This independence of the charge carrier mobilities on the gate-voltage in IDT-BT has been associated to an exceptionally low degree of energetic disorder of the polymer backbone. 

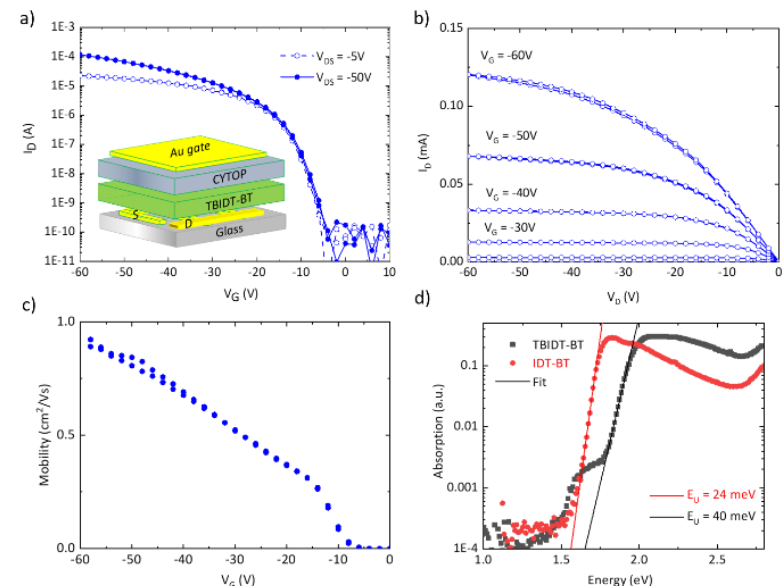

Figure 2. Performance of TBIDT-BT polymer transistors. a) Linear and saturation transfer characteristics as well as b) output characteristics of a representative TBIDT-BT OFET (Length $(\mathrm{L})=20 \mu \mathrm{m}$ and Width $(\mathrm{W})=1 \mathrm{~mm}$ ); the device architecture is shown as an inset in a); c) Extracted saturation charge carrier mobility of a representative TBIDT-BT OFET; d) Absorption of TBIDT-BT and IDT-BT films, measured by Photothermal Deflection Spectroscopy. The solid line represents an exponential tail fit for extraction of the Urbach En$\operatorname{ergy}\left(E_{u}\right)$.

Photothermal deflection spectroscopy (PDS) was used to probe the energetic disorder of TBIDT-BT. It has previously been shown that the optically measured joint density of states (DOS), measured by PDS reflects accurately the electronic sub-bandgap traps and hence, charge transport properties. ${ }^{12,} 17$ Figure $2 \mathrm{~d}$ shows the measured PDS spectrum of TBIDT-BT and IDT-BT as well as a fit to the sub-bandgap tail used to extract the Urbach Energy. TBIDT-BT has a comparatively shallow sub-bandgap tail with an Urbach Energy of $40 \mathrm{meV}$, in comparison to the extremely low $24 \mathrm{meV}$ extracted for IDTBT. The higher energetic disorder of TBIDT-BT exceeds the thermal energy $\mathrm{k}_{\mathrm{B}} \mathrm{T}\left(\mathrm{k}_{\mathrm{B}}\right.$, Boltzmann constant). Hence high carrier concentrations populate more mobile states in the density of states, this could be the reason for the observed gate-voltage dependence of the charge carrier mo-bility. We also observe an extended charge transfer feature in the sub-bandgap absorption of TBIDT-BT, a feature that has previously associated with interchain charge transfer states. ${ }^{14}$ This might also explain why the polymer despite its relatively large Urbach energy of $40 \mathrm{meV}$ can still achieve respectable hole mobilities approaching $1 \mathrm{~cm}^{2} \mathrm{~V}^{-1} \mathrm{~s}^{-1}$.

Scanning Tunneling Microscopy (STM) measurements were performed under ultrahigh vacuum conditions on sub-monolayer films of TBIDT-BT and IDT-BT deposited on a Au(111) surface by electrospray deposition (ESD), according to a recently developed methodology (see SI). ${ }^{18}$ Figure za shows a large scan STM image where individual TBIDT-BT polymer strands appear as worm-like chains with regularly spaced bright protrusions (occasional even brighter protrusion appear where polymer strands cross). The image demonstrates the absence of long-range order in the ESD films and the high flexibility of the polymers resulting in several high-curvature bends. On the local scale, the polymer strands tend, however, to align parallel to each other, as can be seen in the higher magnification image of Figure $3 \mathrm{~b}$. The polymer backbones are clearly recognized, demonstrating that the regular bright protrusions are actually double-dot features periodically separated by $2.7 \pm 0.3 \mathrm{~nm}$. This distance is very close to the length of the repeat unit of TBIDT-BT as calculated by DFT (2.57 $\mathrm{nm}$ ). The alkyl side chains can also be identified as slightly less bright linear features oriented almost perpendicular to the backbone. By superposing a molecular model of TBIDT-BT on the STM images (Figure 3b), it becomes evident that the alkyl chains interdigitate in these closely packed regions. The model also allows the identification of the bright double-dot features as being related to the two non-planar $\mathrm{sp}^{3}$ bridging carbons in the TBIDT unit (one on each side of the backbone) and the determination of the orientation of individual TBIDT units along the backbone.
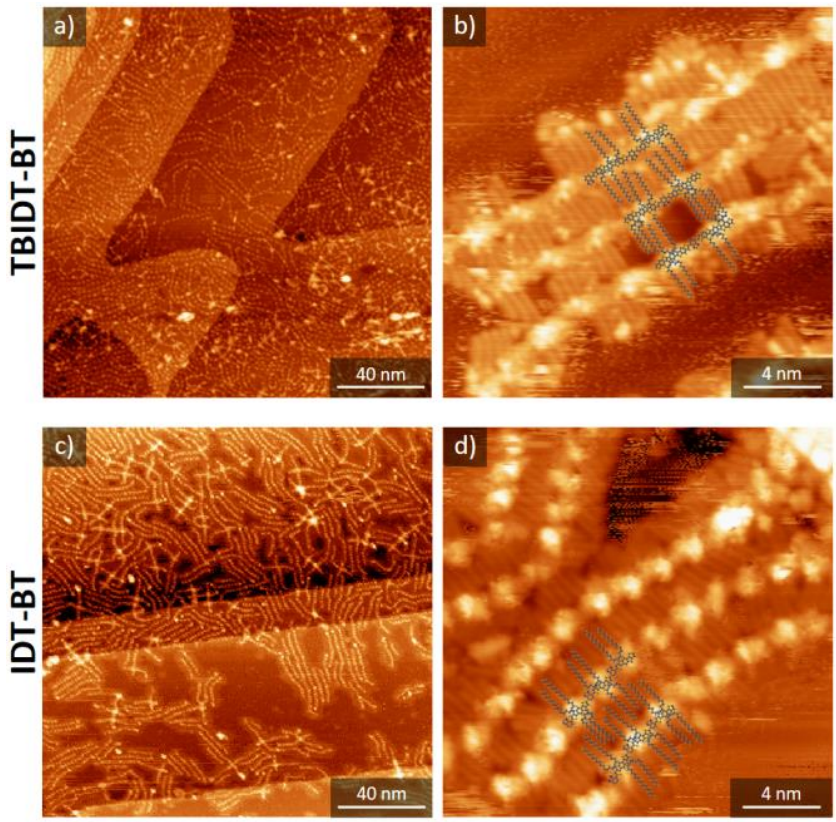

Figure 3. STM analysis of TBIDT-BT and IDT-BT deposited by ESD on $\mathrm{Au}(111)$. a) Large scale STM image of TBIDT-BT, sample bias $\mathrm{V}=-1.4 \mathrm{~V}$, tunneling current $\mathrm{I}=70 \mathrm{pA}$. b) Corresponding higher magnification image $(\mathrm{V}=-0.8 \mathrm{~V}, \mathrm{I}=110 \mathrm{pA})$. c) Large scale STM image of IDT-BT (V=-1.5 V, I = 30 pA). d) Corresponding higher magnification image $(\mathrm{V}=-1.3 \mathrm{~V}, \mathrm{I}=70$ $\mathrm{pA})$. Molecular models of the corresponding polymers are superposed in b) and d).

The analysis of a larger set of STM data for TBIDT-BT films (see $\mathrm{S}_{5}$ in the Supplementary Information) revealed that while the orientation of consecutive TBIDT units within a given backbone is essentially random, TBIDT units in adjacent locally parallel backbones generally have the same orientation as one another. This configuration maximizes van der Waals (vdW) contact between the side chains but is more difficult to achieve because it is statistically less frequent. Even in these more compact assemblies, nanovoids are observed in the alkyl chain regions in between polymer backbones (e.g. in the center of Figure $3 \mathrm{~b}$ ).

The STM image in Figure 3 c shows that IDT-BT deposited on $\mathrm{Au}(111)$ has a similar appearance to TBIDT-BT when imaged on a large scale, with polymers strands looking as elongated 
chains, dotted with a regular sequence of bright protrusions. Also in this case we assign these latter to the two non-planar $\mathrm{sp}^{3}$ bridging carbons in the IDT units. The average distance between the bright protrusions is $1.6 \pm 0.1 \mathrm{~nm}$, in excellent agreement with the theoretically predicted periodicity IDTBT. In higher magnification images such as Figure $3 d$ it is possible to resolve the alkyl side chains and to recognize that they interdigitate where the polymer backbones are locally oriented parallel to each other. At variance with the case of TBIDT-BT, these closely packed regions are much less defective for IDT-BT and do not present any nanovoids.

A simple molecular modelling demonstrates this effect and highlights the importance of the side-chain density for optimal polymer packing. Figure 4 compares the $2 \mathrm{D}$ assembly of TBIDT-BT and IDT-BT oligomers considering the case where the sidechain-bearing units of the oligomers are oriented identically to each other (left column in Figure 4) and the case where these units have a different orientation (right column). The orientation of the TBIDT and IDT subunits can be identified according to the red or purple color of the pairs of dots that have been superposed onto their two non-planar $\mathrm{sp}^{3}$ bridging carbons. It is evident that, for both polymers, the highest ordered assemblies are obtained when the sidechainbearing units have the same orientation. In such cases the vdW contact is maximized throughout the assembly; this is also what has been often observed experimentally for TBIDTBT, as shown in the Supplementary Information. However it should be noted that, because of its higher sidechain attachment density, IDT-BT is capable of efficient interdigitation also in cases where the sidechain-bearing units have different orientations (Figure 4d), managing to achieve an excellent overall vdW contact, which is seen also experimentally (Figure 3d). This ability is significantly reduced for TBIDT-BT (Figure 4b) which has a lower side-chain attachment density, resulting in some of the sidechains to be isolated and causing the formation of nanovoids even in the parallel orientation case (Figure $4 \mathrm{a}$ ). This can lead to a number of problems in the packing of TBIDT-BT, in comparison to IDT-BT, such as a higher chance for possible assembly errors and a lower energy gain (via vdW contacts) per unit length. An additional issue of the nanovoids present in TBIDT-BT is the opportunity for the insertion of impurities, thereby disrupting the assembly, which can be seen in the STM images (Figure $3 \mathrm{~b}$ and in more detail in Figure S6. While this model is strictly valid in $2 \mathrm{D}$, similar considerations are expected to hold also for the ${ }_{3} \mathrm{D}$ assembly of thin films made of these polymers. Both effects lower energy gain from polymer assembly and formation of nanovoids - lead to a less ordered microstructure in TBIDTBT than that observed for IDT-BT, which is likely to be detrimental to the performance of OFET devices based on such thin films, particularly if the free volume within the voids is occupied by species capable of trapping charge carriers, such as water. ${ }^{19}$
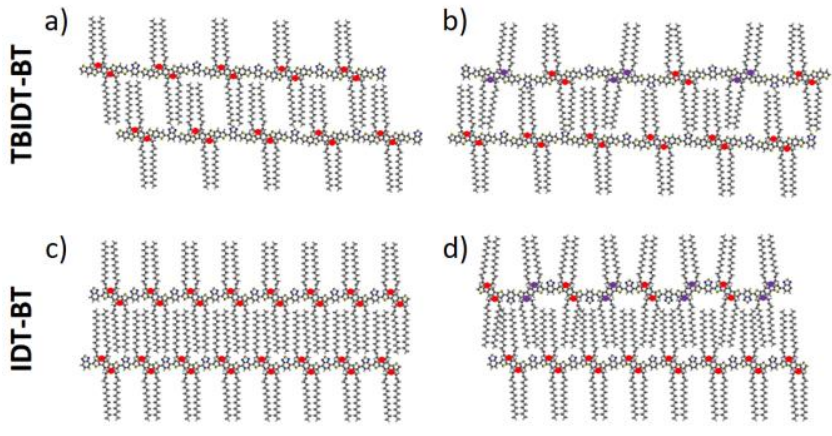

Figure 4. Schematic modelling of the $2 \mathrm{D}$ assembly of TBIDTBT, top row, and IDT-BT, bottom row, oligomers. The red or purple pairs of dots identify different orientations of the TBIDT and IDT subunits.

Some insight into torsional disorder of the polymer backbones can be gained from the calculated potential energy surface (PES) for rotation between the thienyl group with the adjacent BT group (Figure 5). Despite similar non-covalent interactions between atoms close in space, primarily $\mathrm{S}-\mathrm{N}$ or $\mathrm{H}-\mathrm{N}$ interactions, the profiles of IDT-BT and TBIDT-BT display a number of differences to one another. Firstly, the barrier to rotation is lower in TBIDT-BT or, in other words, the planar conformations are less stabilized than in IDT-BT. The consequence of this might be a greater degree of disorder along the polymer chain. Secondly, the energetic difference between the more stable $180^{\circ}$ conformer and the less favored $o^{\circ}$ conformer is larger for TBIDT-BT (1.6 kcal/mol) than for IDT-BT (1.0 $\mathrm{kcal} / \mathrm{mol}$ ). Furthermore, the torsional potentials are significantly different to a simple thiophene-BT link (T-BT), which is often used as a model system to explore non-covalent interactions.

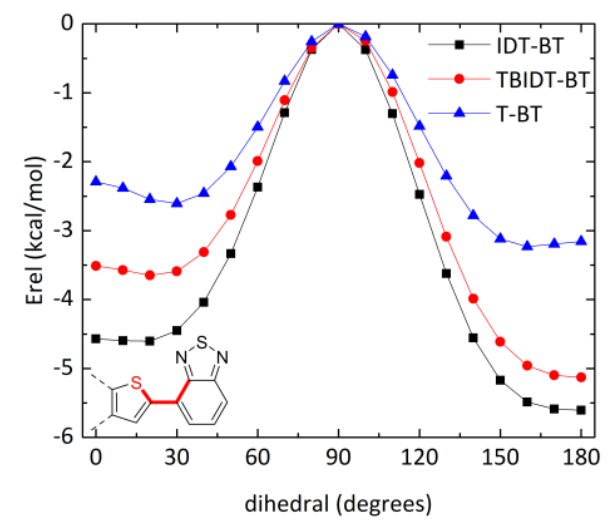

Figure 5. B3LYP-D3/6-31G* Torsional PES of thieno-BT derivatives relative to the energy of the orthogonal conformer, where conjugation and non-covalent interactions are minimal. Rotatable bond highlighted in red, where the $0^{\circ}$ conformer is shown.

Contributing factors to the differences in torsional PES were investigated by comparing atomic charges on each of the atoms contributing to the non-covalent interactions. Mulliken charges from each of the restrained geometry relaxations were extracted for the sulfur and proton of the IDT/TBIDT group, 
and the nitrogen and proton of the BT group (Figure 6a). These were seen to vary with dihedral angle as the electronic structure of the system changes due to increased conjugation and to the changing of interatomic distances. The main difference between TBIDT and IDT groups is the partial charge on sulfur, which is less positive in TBIDT-BT owing to the more electron rich nature, and greater delocalization, of the extended TBIDT group. The positive charge on sulfur is in part due to the delocalization of its lone pair that gives thiophene its aromaticity. From natural bond orbital (NBO) calculations, discussed in more detail below, this delocalization is weaker in the case of TBIDT compared to IDT (see Figure S8). The more localized sulfur lone pair in TBIDT results in a smaller sulfur positive charge. This influences the mostly electrostatic non-covalent interactions during rotation. At $\mathrm{o}^{\circ}$, the attractive $\mathrm{S}^{\delta+}-\mathrm{N}^{\delta-}$ interaction is weaker for TBIDT-BT than IDT-BT, while the $\mathrm{H}^{\delta+}-\mathrm{H}^{\delta+}$ interaction might be marginally more repulsive. At $180^{\circ}$, the repulsive $\mathrm{S}^{\delta+}-\mathrm{H}^{\delta+}$ interaction decreases in TBIDT-BT due to less positive charge on sulfur, while the stabilizing force between $\mathrm{N}_{\mathbf{B T}}$ and $\mathrm{H}_{\text {IDT }}$ remains comparable to that of IDT-BT. These observations may help to explain why TBIDT-BT has a greater preference for the $180^{\circ}$ conformer than the $\mathrm{o}^{\circ}$ conformer.
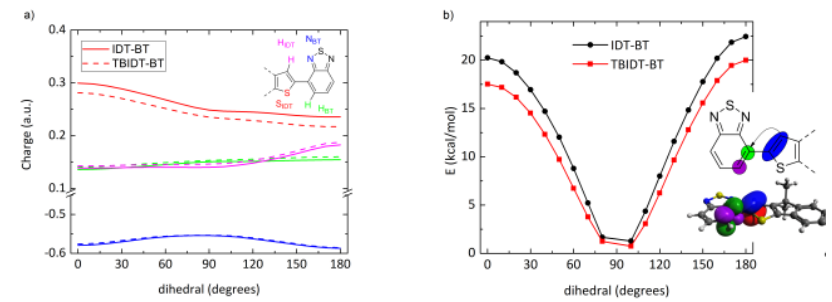

Figure 6. a) Mulliken atomic charges on atoms involved in non-covalent interactions ( $\mathrm{S}_{\text {IDT }}, \mathrm{H}_{\text {IDT }}, \mathrm{N}_{\mathrm{BT}}, \mathrm{H}_{\mathrm{BT}}$ ) as a function of dihedral angle. b) NBO resonance stabilization due to $\pi-\pi^{*}$ donation across the rotatable thieno-BT bond, as a function of dihedral angle.

Information about the difference in barrier height between IDT-BT and TBIDT-BT can be elucidated from the electron delocalization in NBO calculations. These calculations partition electron density into different types of orbitals based on bonding, anti-bonding, and lone pairs on each atom. Resonance stabilization energies for the delocalization of electrons of the $\pi$ bond at the end of the IDT group into the $\pi^{*}$ antibonding orbital of the adjacent BT group are plotted in Figure $6 \mathrm{~b}$. At $90^{\circ}$, there is no orbital overlap and so the resonance stabilization is zero. Upon rotation in either direction, the $\pi$ and $\pi^{*}$ orbitals start to overlap, resulting in increasing stabilization. Rotating to the $180^{\circ}$ conformer results in more efficient orbital overlap than rotating to $\mathrm{o}^{\circ}$, due to the trans relationship between the orbitals. There is a greater stabilization in IDT-BT than in TBIDT-BT of up to $2.5 \mathrm{kcal} / \mathrm{mol}$, resulting in the lower energetic minima in the torsional PES in Figure 5 .
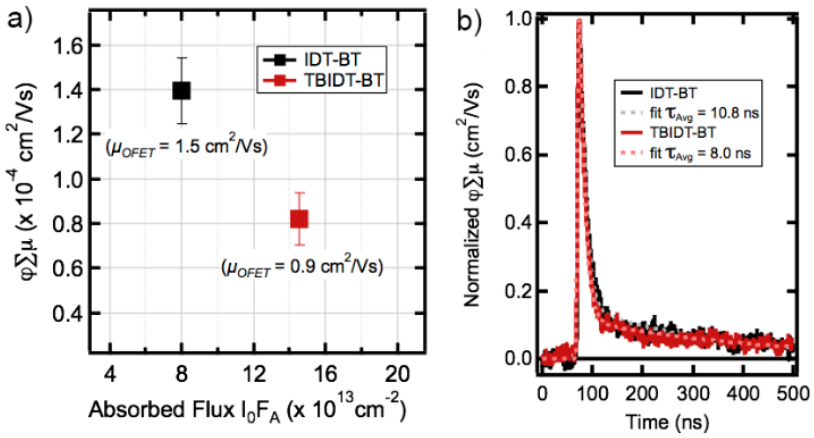

Figure 7. a) Peak $\varphi \Sigma \mu$ values for IDT-BT and TBIDT-BT versus absorbed photon flux using excitation wave-lengths of 640 and $580 \mathrm{~nm}$, respectively. In parentheses, the OFET carrier mobilities are given for reference for each polymer. b) The corresponding normalized transient data recorded over 500 ns with average carrier lifetimes extracted from bi-exponential fits (dashed lines) of the data.

Time-resolved microwave conductivity (TRMC) provides insight into the carrier mobilities measured at the microwave fre-quency of $\sim 9 \mathrm{GHz}$. Thin films of the two polymers were spin-coated onto quartz substrates and excited using a pulsed laser to generate microwave transients, as shown in Figure $7 \mathrm{~b}$ with both exhibiting very similar transient profiles and average carrier lifetimes. Although the exact mechanism for carrier generation is not known, the similar transient profiles suggest that the yield of carriers in both systems is very small, but similar. Therefore, the peak of the transient signal can be used as a good comparison of the carrier mobilities between the two samples. These two peak values of $\phi \Sigma \mu$ are plotted in Figure $7 a$ and exhibit a similar factor of two difference between the two polymer mobilities found by the OFET measurements. Unlike the OFET measurements, however, it should be noted that the mobilities implied by the microwave data are local mobilities and not transport mobilities.

\section{CONCLUSIONS}

In summary, we have designed and synthesised a novel TBIDT moiety with linear alkyl chain substitution, using a novel acid promoted cascade cyclization strategy, which could be used in the preparation of high-mobility transistor materials with rigid backbones. Upon copolymerization of TBIDT with benzothiadiazole, the polymer TBIDT-BT was prepared. Surprisingly, the energetic disorder of this large ring polymer was higher than the benchmark IDT-BT. On further evaluation of the polymer microstructure, defects in the side chain packing assembly were observed by high resolution STM, arising from conformational asymmetry in the side chain distribution and an irregular interdigitated packing density. Consequently, the lower side-chain density of TBIDT-BT, in comparison to IDT-BT, leads to fewer vdW contacts and the presence of nanovoids, thereby disrupting its packing and resulting in less-ordered thin-films. Moreover, torsional PES and atomic charge calculations further highlighted a further potential cause for lower polymer backbone order in TBIDT-BT, owing to the lower energetic barriers to rotation about the TBIDT-BT bond. BC/TG field-effect transistors fabricated from TBIDT-BT yielded a p-type mobility of $0.9 \mathrm{~cm}^{2} \mathrm{~V}^{-1} \mathrm{~s}^{-1}$, which is lower than that of IDT-BT 
$\left(1.5 \mathrm{~cm}^{2} \mathrm{~V}^{-1} \mathrm{~s}^{-1}\right)$. These results demonstrate that the regularity of side chain density, independent of conformational differences along the backbone, as well as the optimization of noncovalent interactions for backbone planarity are both critical to achieve high charge carrier mobility. These findings provide new insight into the fundamental requirements to design high charge carrier mobility semiconducting polymers.

\section{ASSOCIATED CONTENT}

This material is available free of charge via the Internet at http://pubs.acs.org." Experimental details, synthesis of polymers, device fabrication, and detailed experimental procedures.

\section{AUTHOR INFORMATION}

\section{Corresponding Author}

* hu.chen@kaust.edu.sa

*andrew.wadsworthı@imperial.ac.uk

\section{Author Contributions}

The manuscript was written through contributions of all authors. All authors have given approval to the final version of the manuscript. H. Chen and A. Wadsworth contributed equally and share first authorship.

\section{ACKNOWLEDGMENT}

The authors thank KAUST and BASF for financial support and acknowledge EC FP7 Project SC2 (610115) EC H2O2O
(643791), and Engineering and Physical Sciences Research Council (EPSRC) Projects EP/G037515/1 and EP/Moo5143/1. M.N. and H.S. acknowledge financial support from the EPSRC though a Programme Grant (EP/Moo5141/1). G.C. acknowledges financial support from the University of Warwick. AS and CC gratefully acknowledge financial support from the National Science Foundation, Division of Materials Research Award\# 1808401. Use of the Stanford Synchrotron Radiation Lightsource, SLAC National Accelerator Laboratory, is supported by the U.S. Department of Energy, Office of Science, Office of Basic Energy Sciences under Contract No. DE-ACo2-76SFoo515. The transient microwave conductivity work was funded from the through the Solar Photochemistry Program of Office of Basic Energy Sciences. This work was authored in part by the National Renewable Energy Laboratory, operated by Alliance for Sustainable Energy, LLC, for the U.S. Department of Energy (DOE) under Contract No. DE-AC36-o8GO28308. The views expressed in the article do not necessarily represent the views of the DOE or the U.S. Government. The U.S. Government retains and the publisher, by accepting the article for publication, acknowledges that the U.S. Government retains a nonexclusive, paidup, irrevocable, worldwide license to publish or reproduce the published form of this work, or allow others to do so, for U.S. Government purposes.

\section{REFERENCES}


(1) Anthony, J. E.; Brooks, J. S.; Eaton, D. L.; Parkin, S. R. Functionalized Pentacene: Improved Electronic Properties from Control of Solid-State Order. J. Am. Chem. Soc. 2oo1, 123, 9482.

(2) Takimiya, K.; Ebata, H.; Sakamoto, K.; Izawa, T.; Otsubo, T.; Kunugi, Y. 2,7-Diphenyl[1]benzothieno[3,2b]benzothiophene, A New Organic Semiconductor for AirStable Organic Field-Effect Transistors with Mobilities up to $2.0 \mathrm{~cm}^{2} \mathrm{~V}^{-1} \mathrm{~s}^{-1}$. J. Am. Chem. Soc. 2006, 128, 12604.

(3) McCulloch, I.; Heeney, M.; Bailey, C.; Genevicius, K.; MacDonald, I.; Shkunov, M.; Sparrowe, D.; Tierney, S.; Wagner, R.; Zhang, W.; Chabinyc, M. L.; Kline, R. J.; McGehee, M. D.; Toney, M. F. Liquid-Crystalline Semiconducting Polymers with High Charge-Carrier Mobility. Nat. Mater. 2006, 5, 328.

(4) Stalder, R.; Mei, J.; Graham, K. R.; Estrada, L. A.; Reynolds, J. R. Isoindigo, a Versatile Electron-Deficient Unit For High-Performance Organic Electronics. Chem. Mater. 2014, 26, 664 .

(5) Naber, R. C. G.; Tanase, C.; Blom, P. W. M.; Gelinck, G. H.; Marsman, A. W.; Touwslager, F. J.; Setayesh, S.; de Leeuw, D. M. High-Performance Solution-Processed Polymer Ferroelectric Field-Effect Transistors. Nat. Mater. 2005, 4, 243.

(6) Smith, J.; Zhang, W.; Sougrat, R.; Zhao, K.; Li, R.; Cha, D.; Amassian, A.; Heeney, M.; McCulloch, I.; Anthopoulos, T. D. Solution - Processed Small Molecule - Polymer Blend Organic Thin - Film Transistors with Hole Mobility Greater than $5 \mathrm{~cm}^{2} /$ Vs. Adv. Mater. 2012, 24, 2441.

(7) Paterson, A. F.; Treat, N. D.; Zhang, W.; Fei, Z.; WyattMoon, G.; Faber, H.; Vourlias, G.; Patsalas, P. A.;

Solomeshch, O.; Tessler, N.; Heeney, M.; Anthopoulos, T. D. Small Molecule/Polymer Blend Organic Transistors with Hole Mobility Exceeding $13 \mathrm{~cm}^{2} \mathrm{~V}^{-1} \mathrm{~s}^{-1}$. Adv. Mater. 2016, 28, 7791.

(8) Würthner, F.; Stolte, M. Naphthalene and Perylene Diimides for Organic Transistors. Chem. Commun. 2011, 47, 5109.

(9) Anthony, J. E. The Larger Acenes: Versatile Organic Semiconductors. Angew. Chem. Int. Ed. 2008, 47, 452. (10) Zhang, X.; Bronstein, H.; Kronemeijer, A. J.; Smith, J.; Kim, Y.; Kline, R. J.; Richter, L. J.; Anthopoulos, T. D.; Sirringhaus, H.; Song, K.; Heeney, M.; Zhang, W.; McCulloch, I.; DeLongchamp, D. M. Molecular Origin of High Field-Effect Mobility in an IndacenodithiopheneBenzothiadiazole Copolymer. Nat. Commun. 2013, 4, 2238.
(11) Zhang, W.; Smith, J.; Watkins, S. E.; Gysel, R.; McGehee, M.; Salleo, A.; Kirkpatrick, J.; Ashraf, S.; Anthopoulos, T.; Heeney, M.; McCulloch, I.

Indacenodithiophene Semiconducting Polymers for HighPerformance, Air-Stable Transistors. J. Am. Chem. Soc. 2o1o, 132, 11437.

(12) Venkateshvaran, D.; Nikolka, M.; Sadhanala, A.; Lemaur, V.; Zelazny, M.; Kepa, M.; Hurhangee, M.; Kronemeijer, A. J.; Pecunia, V.; Nasrallah, I.; Romanov, I.; Broch, K.; McCulloch, I.; Emin, D.; Olivier, Y.; Cornil, J.; Beljonne, D.; Sirringhaus, H. Approaching Disorder-Free Transport in High-Mobility Conjugated Polymers. Nature 2014, 515, 384 .

(13) Chen, H.; Hurhangee, M.; Nikolka, M.; Zhang, W.; Kirkus, M.; Neophytou, M.; Cryer, S. J.; Harkin, D.; Hayoz, P.; Abdi-Jalebi, M.; McNeill, C. R.; Sirringhaus, H.; McCulloch, I. Dithiopheneindenofluorene (TIF) Semiconducting Polymers with Very High Mobility in Field - Effect Transistors. Adv. Mater. 2017, 29, 1702523. (14) Thomas, T. H.; Harkin, D. J.; Gillett, A. J.; Lemaur, V.; Nikolka, M.; Sadhanala, A.; Richter, J. M.; Armitage, J.; Chen, H.; McCulloch, I.; Menke, S. M.; Olivier, Y.; Beljonne, D.; Sirringhaus, H. Short Contacts Between Chains Enhancing Luminescence Quantum Yields and Carrier Mobilities in Conjugated Copolymers. Nat. Commun. 2019, 10, 2614 .

(15) McCulloch, I.; Salleo, A.; Chabinyc, M. Avoid the Kinks When Measuring Mobility. Science 2016, 352, 1521.

(16) Paterson, A. F.; Singh, S.; Fallon, K. J.; Hodsden, T.; Han, Y.; Schroeder, B. C.; Bronstein, H.; Heeney, M.; McCulloch, I.; Anthopoulos, T. D. Recent Progress in High Mobility Organic Transistors: A Reality Check. Adv. Mater. 2018, 30, 1801079 .

(17) Nikolka, M.; Hurhangee, M.; Sadhanala, A.; Chen, H.; McCulloch, I.; Sirringhaus, H. Correlation of Disorder and Charge Transport in a Range of Indacenodithiophene -

Based Semiconducting Polymers. Adv. Electron. Mater. 2018, 4, 1700410.

(18) Warr, D. A.; Perdigão, L. M. A.; Pinfold, H.; Blohm, J.; Stringer, D.; Leventis, A.; Bronstein, H.; Troisi, A.;

Costantini, G. Sequencing Conjugated Polymers by Eye. Sci. Adv. 2018, 4, 9543.

(19) Nikolka, M.; Broch, K.; Armitage, J.; Hanifi, D.; Nowack, P. J.; Venkateshvaran, D.; Sadhanala, A.; Saska, J.; Mascal, M.; Jung, S.-H.; Lee, J. K.; McCulloch, I.; Salleo, A.; Sirringhaus, H. High-Mobility, Trap-Free Charge Transport in Conjugated Polymer Diodes. Nat. Commun. 2019, 10, 2122. 
Insert Table of Contents artwork here
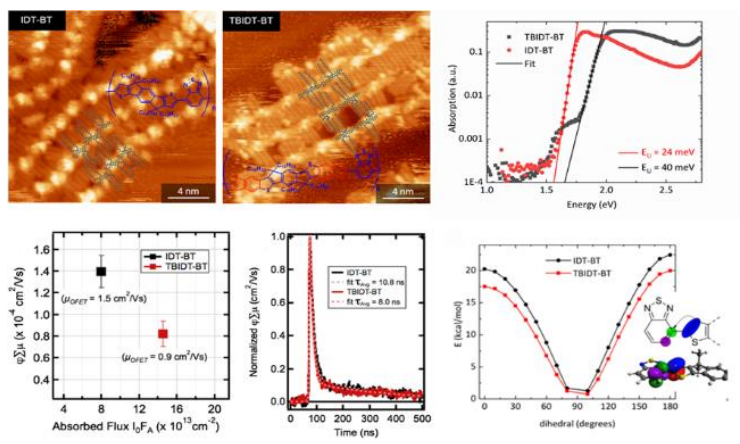